\title{
RESEARCH
}

\section{Best rank-k approximations for tensors: generalizing Eckart-Young}

\author{
Jan Draisma ${ }^{1,2^{*}}$ (D) Giorgio Ottaviani ${ }^{3}$ and Alicia Tocino ${ }^{4}$
}

\author{
*Correspondence: \\ jan.draisma@math.unibe.ch \\ ${ }^{1}$ Mathematisches Institut, \\ Universität Bern, Sidlerstrasse 5, \\ 3012 Bern, Switzerland \\ The first author was partially \\ supported by the NWO Vici grant \\ entitled Stabilisation in Algebra \\ and Geometry. \\ The second author is member of \\ INDAM-GNSAGA. \\ Full list of author information is \\ available at the end of the article
}

\begin{abstract}
Given a tensor $f$ in a Euclidean tensor space, we are interested in the critical points of the distance function from $f$ to the set of tensors of rank at most $k$, which we call the critical rank-at-most-k tensors for $f$. When $f$ is a matrix, the critical rank-one matrices for $f$ correspond to the singular pairs of $f$. The critical rank-one tensors for $f$ lie in a linear subspace $H_{f}$, the critical space of $f$. Our main result is that, for any $k$, the critical rank-at-most-k tensors for a sufficiently general $f$ also lie in the critical space $H_{f}$. This is the part of Eckart-Young Theorem that generalizes from matrices to tensors. Moreover, we show that when the tensor format satisfies the triangle inequalities, the critical space $H_{f}$ is spanned by the complex critical rank-one tensors. Since $f$ itself belongs to $H_{f}$, we deduce that also $f$ itself is a linear combination of its critical rank-one tensors.
\end{abstract}

Keywords: Tensor, Eckart-Young Theorem, Best rank-k approximation

Mathematics Subject Classification: 15A69, 15A18, 14M17, 14P05

\section{Introduction}

The celebrated Eckart-Young Theorem says that, for a real $m \times n$-matrix $A$ with $m \leq n$ and for an integer $k \leq m$, a matrix $B$ of rank at most $k$ nearest to $A$ is obtained from $A$ as follows: Compute the singular value decomposition $A=U \Sigma V^{T}$, where $U, V$ are orthogonal matrices and where $\Sigma=\operatorname{diag}\left(\sigma_{1}, \ldots, \sigma_{m}\right)$ is the "diagonal" $m \times n$ matrix with the singular values $\sigma_{1} \geq \cdots \geq \sigma_{m} \geq 0$ on its main diagonal, and set $B:=U \operatorname{diag}\left(\sigma_{1}, \ldots, \sigma_{k}, 0, \ldots, 0\right) V^{T}$. Such a best rank-k approximation is unique if and only if $\sigma_{k}>\sigma_{k+1}$, and for us "nearest" refers to the Frobenius norm (but in fact, the result holds for arbitrary $\mathrm{O}_{m} \times \mathrm{O}_{n}$-invariant norms [12]).

For higher-order tensors, an analogous approach for finding best rank- $k$ approximations fails in general [18]. It succeeds, with respect to the Frobenius norm, for orthogonally decomposable tensors $[1,18]$, but this is a very low-dimensional real-algebraic variety in the space of all tensors. In this paper, we will establish versions of the Eckart-Young Theorem and the Spectral Theorem that do hold for general tensors.

To motivate this theorem, consider matrices once again, and assume that the $\sigma_{i}$ are distinct and positive. A statement generalizing the Eckart-Young Theorem says that we obtain all critical points of the distance function $d_{A}(B):=\|A-B\|^{2}$ on the manifold of rank- $k$ matrices by setting any $m-k$ of the singular values equal to zero [3], so as to obtain a matrix 


$$
B_{i_{1}, \ldots, i_{k}}:=U \operatorname{diag}\left(0, \ldots, 0, \sigma_{i_{1}}, 0, \ldots, 0, \sigma_{i_{2}}, 0, \ldots, 0, \sigma_{i_{k}}, 0, \ldots\right) V^{T}
$$

for any ordered $k$-tuple $i_{1}<\cdots<i_{k}$ in $\{1, \ldots, m\}$. We will call these critical points critical rank-k matrices for $A$. In particular, the critical rank-one matrices are $B_{1}, \ldots, B_{m}$, and we draw attention to the fact that for each $k \geq 1$ and each $k$-tuple $i_{1}<\cdots<i_{k}$ the critical rank- $k$ matrix $B_{i_{1}, \ldots, i_{k}}$ lies in the linear span of $B_{1}, \ldots, B_{m}$. Moreover, this linear span has a direct description in terms of $A$ : It consists of all matrices $B$ such that both $A^{T} B$ and $A B^{T}$ are symmetric matrices.

Taking cue from this observation, we will associate a critical space $H_{f}$ to a tensor $f$ show that $H_{f}$ contains the critical rank-at-most- $k$ tensors for $f$ for each value of $k$ (see below for a definition), and that $H_{f}$ is spanned by the critical rank-one tensors for $f$. We will establish these results for sufficiently general partially symmetric tensors, and we work over the base field $\mathbb{C}$ rather than $\mathbb{R}$.

Theorem 1.1 Let $f$ be a sufficiently general tensor in $S^{d_{1}} \mathbb{C}^{n_{1}+1} \otimes \cdots \otimes S^{d_{p}} \mathbb{C}^{n_{p}+1}$. Then, for each natural number $k$, the critical rank-at-most- $k$ tensors for $f$ lie in the critical space $H_{f}$. Moreover, if for each $\ell$ with $d_{\ell}=1$ the triangle inequality $n_{\ell} \leq \sum_{i \neq \ell} n_{i}$ holds, then codim $H_{f}=\sum_{\ell}\left(\begin{array}{c}n_{\ell}+1 \\ 2\end{array}\right)$ and $H_{f}$ is spanned by the critical rank-one tensors for $f$. In particular, $f$ itself lies in the linear span of the critical rank-one tensors for $f$.

We record the following two corollaries over the real numbers.

Corollary 1.2 If $n_{1}, \ldots, n_{p}$ satisfy the triangle inequality $n_{\ell} \leq \sum_{i \neq \ell} n_{i}$ for each $\ell=$ $1, \ldots, p$, then for a sufficiently general tensor $f \in \bigotimes_{i=1}^{p} \mathbb{R}^{n_{i}+1}$ and any natural number $k$, any real tensor of real rank at most $k$ closest to $f$ in the Frobenius norm lies in the linear span of the complex critical rank-one tensors for $f$. In particular, $f$ itself lies in the linear span of the complex critical rank-one tensors for $f$.

Corollary 1.3 For a sufficiently general symmetric tensor $f \in S^{d} \mathbb{R}^{n+1}$ and any natural number $k$, any real symmetric tensor of real symmetric rank at most $k$ closest to $f$ in the Frobenius norm lies in the linear span of the complex critical symmetric rank-one tensors for $f$. In particular, $f$ itself lies in the linear span of the complex critical rank-one tensors forf.

In the case of symmetric tensors, these critical rank-one tensors correspond to the socalled eigenvectors of $f$ [11], while in the case of ordinary tensors, they correspond to singular vector tuples [10]. In the case $n=1$ of binary forms, Corollary 1.3 was proved in [16]. The two corollaries above can be regarded as generalizations of the Eckart-Young Theorem and the Spectral Theorem from matrices to tensors.

Several remarks are in order here. First, we complexify $d_{f}$ to the quadratic polynomial $d_{f}(u):=(u-f \mid u-f)$, where (.|.) is the standard complex bilinear form on the space of tensors (and not a Hermitian form). The point of doing this is that, unlike for matrices, the critical points of this function on low-rank tensors are in general not real anymore, even if $f$ is real. Accordingly, the critical space $H_{f}$, while defined by linear equations over $\mathbb{R}$ if $f$ is real, is taken to be the space of complex solutions to those equations. Second, we denote the dimensions by $n+1$ rather than $n$ since we will be using methods from projective algebraic geometry where the formulas look more appealing in terms of the projective dimension $n$ than in the affine dimension $n+1$. An example of this phenomenon is the 
triangle inequalities in the theorem, which hold if and only if the variety dual to the SegreVeronese embedding of the product $\mathbb{P}^{n_{1}} \times \cdots \times \mathbb{P}^{n_{p}}$ via degrees $d_{1}, \ldots, d_{p}$ is a hypersurface [7, Corollary 5.11].

The remainder of this paper is organized as follows. In Sect. 2, we define the critical space $H_{f}$ for a partially symmetric tensor $f$ and prove that the critical rank-at-most- $k$ tensors for $f$ lie in $H_{f}$. Then, in Sect. 3, we use vector bundle techniques to compute the dimension of the space spanned by the critical rank-one tensors for $f$ and to show that this space equals $H_{f}$. In Sect. 4, we combine these ingredients to establish the results above.

\section{The critical space of a tensor}

\subsection{Partially symmetric tensors and their ranks}

Let $p \in \mathbb{Z}_{\geq 1}$, let $V_{1}, \ldots, V_{p}$ be complex vector spaces, and let $d_{1}, \ldots, d_{p} \in \mathbb{Z}_{\geq 1}$. Let $S^{d} V$ be the $d$ th symmetric power of $V$. We will study tensors in the space

$$
T:=S^{d_{1}} V_{1} \otimes \cdots \otimes S^{d_{p}} V_{p} .
$$

So for $p=1, T$ is a symmetric power of $V_{1}$, which is canonically isomorphic with the space of symmetric, $d_{p}$-way $n_{1} \times \cdots \times n_{1}$-tensors. On the other hand, when all $d_{i}$ are equal to 1 , then $T$ is a space of $p$-way ordinary tensors. We will write $[p]:=\{1, \ldots, p\}$.

Inside $T$, let $X$ be the set of all tensors of the form

$$
v_{1}^{d_{1}} \otimes \cdots \otimes v_{p}^{d_{p}} \quad\left(v_{\ell} \in V_{\ell}, \ell \in[p]\right) .
$$

Then, $X$ is a closed subvariety of $T$ known as the affine cone over the Segre-Veronese embedding of $\mathbb{P}^{n_{1}} \times \cdots \times \mathbb{P}^{n_{p}}$ of degrees $\left(d_{1}, \ldots, d_{p}\right)$. Let $k X$ denote the set of sums of $k$ elements of $X$. An arbitrary element $t$ of $T$ lies in $k X$ for some $k$, and the minimal such $k$ is called the rank of $t$ [9, Definition 5.2.1.1]. For $p=1$, this is the symmetric or Waring rank, and if all $d_{q}$ are 1, this notion is ordinary tensor rank. We write $\operatorname{Sec}_{k}(X)$ for the Zariski (or Euclidean) closure of $k X$ in $T$.

For real tensors, a few modifications are needed. The real rank of a real tensor $t$ is the minimum $k$ such that $t=\sum_{i=1}^{k} \lambda_{i} x_{i}$ with $\lambda_{i} \in \mathbb{R}$ and $x_{i} \in X_{\mathbb{R}}$ (it is enough to allow $\lambda_{i}= \pm 1$ ). For example $\left(e_{1}+i e_{2}\right)^{3}+\left(e_{1}-i e_{2}\right)^{3}$ has complex rank 2 and real rank 3 . Real rank is subtle for low-rank approximation of tensors. A classical example of de Silva and Lim [2] shows that for almost all $2 \times 2 \times 2$-tensors of real rank 3 (like the above one) does not exist a closest tensor of real rank 2 , while such phenomena may happen only for measure zero subsets of the set of complex tensors of given rank.

\subsection{Symmetric bilinear forms and pairings}

If $V, W$ are complex vector spaces with symmetric bilinear forms (.|.), and if $d \in \mathbb{Z}_{\geq 0}$, then $S^{d} V$ and $V \otimes W$ carry unique symmetric bilinear forms, also denoted (.|.), that satisfy

$$
\begin{aligned}
\left(v^{d} \mid v^{\prime d}\right) & :=\left(v \mid v^{\prime}\right)^{d} \text { and } \\
\left(v \otimes w \mid v^{\prime} \otimes w^{\prime}\right) & :=\left(v \mid v^{\prime}\right)\left(w \mid w^{\prime}\right) .
\end{aligned}
$$

The first of these equalities implies

$$
\left(v_{1} \ldots v_{d} \mid v^{\prime d}\right)=\prod_{i=1}^{d}\left(v_{i} \mid v^{\prime}\right)
$$


and more in general

$$
\left(v_{1} \cdots v_{d} \mid v_{1}^{\prime} \cdots v_{d}^{\prime}\right)=\frac{1}{d !} \sum_{\pi \in S_{d}} \prod_{i=1}^{d}\left(v_{i} \mid v_{\pi(i)}^{\prime}\right) .
$$

We now fix nondegenerate symmetric bilinear forms on each $V_{\ell}, \ell \in[p]$. Then, iterating these constructions, we obtain a canonical bilinear form on $T$.

Using the bilinear forms on $V$ and $W$, we can also build more general bilinear maps whose outputs are vectors or tensors rather than scalars. We will call these bilinear maps pairings and denote them by [...]. Of particular relevance to us is the skew-symmetric pairing $S^{d} V \times S^{d} V \rightarrow \bigwedge^{2} V$ determined by

$$
\left[v^{d} \mid w^{d}\right]:=(v \mid w)^{d-1} v \wedge w,
$$

which implies

$$
\left[v_{1} \ldots v_{d} \mid w^{d}\right]=\frac{1}{d} \sum_{i^{\prime} \in[d]}\left(\prod_{i \neq i^{\prime}}\left(v_{i} \mid w\right)\right) v_{i^{\prime}} \wedge w
$$

and more in general

$$
\left[v_{1} \cdots v_{d} \mid w_{1} \cdots w_{d}\right]=\frac{1}{d \cdot d !} \sum_{i^{\prime}, j^{\prime} \in[d]} \sum_{\pi:[d] \backslash i^{\prime} \rightarrow[d] \vee^{\prime}}\left(\prod_{i \neq i^{\prime}}\left(v_{i} \mid w_{\pi(i)}\right)\right) v_{i^{\prime}} \wedge w_{j^{\prime}},
$$

where $\pi$ runs over all bijections $[d] \backslash i^{\prime} \rightarrow[d] \backslash j^{\prime}$.

Remark 2.1 In the case of binary forms $(\operatorname{dim} V=2$ and arbitrary $d)$, the pairing $[f \mid g]$ coincides (up to scalar multiples) with $\left(f \mid D(g)\right.$ ), where $D(g)=g_{x} y-g_{y} x$; see [16]. Note the skew-symmetry property $(f \mid D(g))=-(g \mid D(f))$. On the other hand, in the case of symmetric matrices $(d=2$ and arbitrary $V$ ), the pairing $[f \mid g]$ coincides (up to scalar multiples) with the bracket $f g-g f$.

Building on this construction, for each $\ell \in[p]$ we define a pairing [... $]_{\ell}: T \times T \rightarrow \bigwedge^{2} V_{\ell}$ by

$$
\left[f_{1} \otimes \cdots \otimes f_{p} \mid g_{1} \otimes \cdots \otimes g_{p}\right]_{\ell}:=\left(\prod_{i \neq \ell}\left(f_{i} \mid g_{i}\right)\right)\left[f_{\ell} \mid g_{\ell}\right], f_{i}, g_{i} \in S^{d_{i}} V_{i},
$$

which we will use to define the critical space.

Remark 2.2 In the case of matrices $T=V_{1} \otimes V_{2}$, the pairing $[f, g]_{1}$ coincides (up to scalar multiples) with $f g^{t}-g f^{t}$, while $[f, g]_{2}$ is (up to scalar multiples) $f^{t} g-g^{t} f$.

\subsection{Basis, orthogonal basis and monomials}

If $B$ is a basis of $V$, then the degree- $d$ monomials in the elements of $B$ form a basis of $S^{d} V$. Such a basis is orthogonal if $B$ is orthogonal. Hence, if we fix bases (respectively, orthogonal bases) of $V_{1}, \ldots, V_{p}$, then by taking tensor products we obtain a basis (respectively, orthogonal basis) of $T$, whose elements we will call monomials of degree $D:=\sum_{\ell=1}^{p} d_{\ell}$. We will use the word $g c d$ of two such monomials $x, y$ for the highest-degree monomial $z$ such that both $x$ and $y$ can be obtained from $z$ by multiplying $z$ with suitable monomials. 
Example 2.3 If $p=3$ and $V_{1}=V_{2}=V_{3}=\mathbb{C}^{3}$ with the standard bilinear form, and $d_{1}=d_{2}=3$ and $d_{3}=2$, then the $\operatorname{gcd}$ of $\left(e_{1}^{2} e_{2}\right) \otimes\left(e_{1} e_{2} e_{3}\right) \otimes\left(e_{1}^{2}\right)$ and $\left(e_{1} e_{2} e_{3}\right) \otimes\left(e_{3}^{3}\right) \otimes\left(e_{2} e_{3}\right)$ equals $\left(e_{1} e_{2}\right) \otimes\left(e_{3}\right) \otimes(1)$.

Lemma 2.4 For two monomials $f=f_{1} \otimes \cdots \otimes f_{p}, g=g_{1} \otimes \cdots \otimes g_{p}$ in $T$ relative to the same orthogonal bases of $V_{1}, \ldots, V_{p}$ and for $\ell \in[p]$ we have $[f \mid g]_{\ell}=0$ unless $f_{i}=g_{i}$ for all $i \neq \ell$ and $h:=\operatorname{gcd}\left(f_{\ell}, g_{\ell}\right)$ has degree $d_{\ell}-1$; in this case $\operatorname{gcd}(f, g)$ has degree $D-1$ and $[u \mid v]_{\ell} \in \mathbb{C}^{*}\left(f_{\ell} / h\right) \wedge\left(g_{\ell} / h\right)$.

This is immediate from the definition of the pairing in (1).

\subsection{Critical rank-one tensors}

Let $f \in T$. Then, the critical points of the distance function $d_{f}: x \mapsto(f-x \mid f-x)$ on $X$ are by definition those $x \in X \backslash\{0\}$ for which $f-x$ is perpendicular to the tangent space $T_{x} X$ to $X$ at $x$; we write this as $f-x \perp T_{x} X$. We call these tensors the critical rank-one tensors for $f$. For sufficiently general $f$, each of these critical rank-one tensors is non-isotropic, i.e., satisfies $(x \mid x) \neq 0$ (see [4, Lemma 4.2], in next Proposition 2.6 we will prove a slightly more general fact).

We will establish a bilinear characterization of these critical rank-one tensors for $f$. First, we describe the tangent space of $X$ at a point $x$ in more detail. For this, write

$$
x=v_{1}^{d_{1}} \otimes \cdots \otimes v_{p}^{d_{p}}
$$

Hence, we may extend each $v_{\ell}$ to a basis of $V_{\ell}$. We then obtain an $x$-adapted basis of $T$ consisting of monomials. If moreover $x$ is non-isotropic, we have $\left(v_{\ell} \mid v_{\ell}\right) \neq 0$ and we may extend each $v_{\ell}$ to an orthogonal basis. We then obtain an $x$-adapted orthogonal basis of $T$.

Lemma 2.5 Let $x \in X$ as in (2).

(1) Then, relative to any $x$-adapted basis, $T_{x} X$ is spanned by all degree-D monomials whose gcd with $x$ has degree at least $D-1$.

(2) Assume moreover that $x$ is non-isotropic. Then, relative to any $x$-adapted orthogonal basis, $\left(T_{x} X\right)^{\perp}$ is spanned by all degree-D monomials whose gcd with $x$ has degree at most $D-2$.

Proof Part (1) follows by applying the Leibniz rule to the parameterization (2) of $X$; part (2) is a straightforward consequence.

Proposition 2.6 Let $f \in T$ and let $x \in X$ be non-isotropic. Then, the following two statements are equivalent:

(1) some (nonzero) scalar multiple of $x$ is a critical rank-one tensor for $f$ and

(2) a unique (nonzero) scalar multiple of $x$ is a critical rank-one tensor for $f$;

and they imply the following statement:

(3) for each $\ell \in[p],[f \mid x]_{\ell} \in \bigwedge^{2} V_{\ell}$ is zero.

Moreover, iff is sufficiently general, then every nonzero $x \in X$ satisfying (3) is non-isotropic and satisfies (1) and (2). 
The pairing in item (3) is the pairing from (1).

Proof For the equivalence of the first two statements, we note that if $c x, c^{\prime} x$ with $c, c^{\prime} \neq 0$ are critical rank-one tensors for $f$, then $T_{c x} X=T_{c^{\prime} x} X=T_{x} X$ and $f-c x \perp T_{x} X$ and $f-c^{\prime} x \perp T_{x} X$. Since $x \in T_{x} X$, we find that $\left(c-c^{\prime}\right) x \perp x$, and using that $x$ is non-isotropic we find that $c=c^{\prime}$.

To prove that (1) implies (3), write $x$ as in (2) and extend each $v_{\ell}$ to an orthogonal basis of $V_{\ell}$, so as to obtain an $x$-adapted orthogonal basis of $T$. Now assume that $f-c x \perp T_{x} X$. Then, by Lemma $2.5, f-c x$ is a linear combination of degree- $D$ monomials whose gcds with $x$ have degrees at most $D-2$. Hence by Lemma $2.4,[x \mid f-c x]_{\ell}=0$. By the skewsymmetry, $[x \mid x]_{\ell}=0$, so $[x \mid f]_{\ell}=0$.

For the last statement, consider an $x=v_{1}^{d_{1}} \otimes \cdots \otimes v_{p}^{d_{p}} \in X$ where, say, $v_{1}, \ldots, v_{a}$ with $a>0$ are isotropic but the remaining factors are not. Extend each $v_{\ell}, \ell>a$ to an orthogonal basis of $V_{\ell}$, and for $v_{\ell}$ with $\ell \leq a$ find an isotropic $w_{\ell} \in V_{i}$ with $\left(v_{\ell} \mid w_{\ell}\right)=1$ and extend $v_{\ell}, w_{\ell}$ with an orthogonal basis of the orthogonal complement of $\left\langle v_{\ell}, w_{\ell}\right\rangle^{\perp}$ to a basis of $V_{\ell}$. In the corresponding (non-orthogonal) monomial basis of $T$, the monomials $y$ with $[y \mid x]_{\ell} \neq 0$ for $\ell \leq a$ are those of the form

$$
w_{1}^{d_{1}} \otimes \cdots \otimes w_{\ell}^{d_{\ell}-1} u_{\ell} \otimes \cdots \otimes w_{a}^{d_{a}} \otimes v_{a+1}^{d_{a+1}} \otimes \cdots \otimes v_{p}^{d_{p}},
$$

where $u_{\ell}$ is a basis vector of $V_{\ell}$ that is distinct from $v_{\ell}$ but possibly equal to $w_{\ell}$. These monomials all satisfy $[y \mid x]_{i}=0$ for $i \neq \ell$. Similarly, the monomials $y$ with $[y \mid x]_{\ell} \neq 0$ for $\ell>a$ are those of the form

$$
w_{1}^{d_{1}} \otimes \cdots \otimes w_{a}^{d_{a}} \otimes v_{a+1}^{d_{a+1}} \otimes \cdots \otimes v_{l}^{d_{l}-1} u_{l} \otimes \cdots \otimes v_{p}^{d_{p}}
$$

with $u_{\ell}$ a basis vector of $V_{\ell}$ distinct from $v_{\ell}$; they, too, satisfy $[y \mid x]_{i}=0$ for $i \neq \ell$. The remaining monomials span the space of $f \mathrm{~s}$ with $[x \mid f]_{\ell}=0$ for all $\ell$; this space therefore has dimension

$$
\operatorname{dim} T-\left(n_{1}+\cdots+n_{p}\right),
$$

and it does not change when we scale $x$. Since the isotropic projective points $\langle x\rangle \in \mathbb{P} T$ form a subvariety of positive codimension in the $\left(n_{1}+\cdots+n_{p}\right)$-dimensional projective variety $\mathbb{P} X$, the locus of all $f$ for which there is a nonzero isotropic $x \in X$ with $[f \mid x]_{\ell}=0$ for all $\ell$ has dimension less than $\operatorname{dim} T$.

Now assume that $f$ is sufficiently general and let $x \in X \backslash\{0\}$ satisfy $[x \mid f]_{\ell}=0$ for all $\ell$. By the above, $x$ is non-isotropic. Suppose that $f$, expanded on the $x$-adapted orthogonal basis, contains a monomial $y$ whose gcd with $x$ has degree exactly $D-1$. If $y$ agrees with $x$ except in the factor $S^{d_{\ell}} V_{\ell}$ where it equals $v_{\ell}^{d_{\ell}-1} u_{\ell}$, then in $[x \mid f]_{\ell}$, expanded on the standard basis of $\wedge^{2} V_{\ell}$ relative to the chosen basis of $V_{\ell}$, the term $v_{\ell} \wedge u_{\ell}$ has a nonzero coefficient. Hence, $[x \mid f]_{\ell}$ is nonzero, a contradiction.

Therefore, $f$ contains only monomials whose gcds with $x$ have degrees at most $D-2$, and possibly the monomial $x$ itself. Then, $f-c x \perp T_{x} X$ for a unique constant $c$. By generality of $f$, it does not lie in $\left(T_{x} X\right)^{\perp}$ for any $x \in X \backslash\{0\}$ (the union of these orthogonal complements is the cone over the variety dual to the projective variety defined by $X$, and of positive codimension). Hence, $c \neq 0$, and $c x$ is a critical rank-one tensor for $f$.

Remark 2.7 The implication (1) $\Longrightarrow$ (3) in Proposition 2.6 holds without the assumption of non-isotropy of $x$. This follows from the fact that the ED correspondence

$$
\{(x, f) \in X \times V \mid x \text { is critical for } f\}
$$


is a irreducible variety (see [3, $\$ 4$ and Lemma 2.1$]$ ) and the nonempty open part in it where $x$ is non-isotropic lies in the variety defined by $[f \mid x]_{\ell}=0 \forall \ell \in[p]$ by Proposition 2.6.

\subsection{The critical space}

In view of Proposition 2.6, we introduce the following notion.

Definition 2.8 For a tensor $f \in T$, the critical space $H_{f} \subseteq T$ off is defined as

$$
H_{f}:=\left\{g \in T \mid[f \mid g]_{\ell}=0 \text { for all } \ell \in[p]\right\} .
$$

Remark 2.9 By the skew-symmetry, it follows immediately that $f \in H_{f}$.

Remark 2.10 In the case of binary forms ( $\operatorname{dim} V=2), H_{f}$ is the hyperplane orthogonal to $D(f)$ [16]. In the case of ordinary tensors, $H_{f}$ was first defined in [15] where it was called singular space, but in view of the results in this paper we feel that critical space is a better name.

Proposition 2.6 establishes that the non-isotropical critical rank-one tensors all lie inside $H_{f}$; hence for a sufficiently general $f$, all critical rank-one tensors lie in $H_{f}$. In the next subsection, we will establish an analogous statement for higher ranks.

Note that the number of linear conditions for $g$ to lie in $H_{f}$ is at most $\sum_{\ell=1}^{p} \operatorname{dim} \wedge^{2} V_{\ell}=$ $\sum_{\ell=1}^{p}\left(\begin{array}{c}n_{\ell}+1 \\ 2\end{array}\right)$-the linear conditions in the definition may not all be linearly independent. In Proposition 3.6 we will see that, assuming the triangle inequalities from Theorem 1.1 and assuming that $f$ is sufficiently general, equality holds.

\subsection{Higher rank}

We will now establish a generalization of Proposition 2.6 to higher-rank tensors.

Definition 2.11 Let $f \in T$ and let $k$ be any nonnegative integer. A critical rank-at-most-k tensor for $f$ is a tensor $g \in k X$ such that $f-g \perp T_{g} \operatorname{Sec}_{k}(X)$.

Note that by [4, Lemma 4.2], all the critical rank-at-most- $k$ tensors for a sufficiently general $f \in T$ are smooth points of $\operatorname{Sec}_{k}(X)$ and can be written as a sum of $k$ nonisotropic rank-one tensors. Moreover, if we assume that $k$ is at most the generic rank of tensors in $T$, then these critical tensors to a sufficiently general $f$ have rank equal to $k$. If $k$ is at least the generic rank of tensors in $T$, then the only critical rank-at-most- $k$ tensor for a sufficiently general $f$ is $f$ itself.

Proposition 2.12 Let $f \in T$ be sufficiently general and let $k$ be a nonnegative integer. Then, all the critical rank-at-most-k tensors for $f$ lie in the critical space $H_{f}$.

Proof Let $g$ be a critical rank-at-most- $k$ tensor. By generality of $f, g$ can be written as $x_{1}+\cdots+x_{k}$ with each $x_{i} \in X$ non-isotropic. Then, $T_{g} \operatorname{Sec}_{k} X \supseteq \sum_{i=1}^{k} T_{x_{i}} X$, so that for each $i \in[k]$ we have $f-g \perp T_{x_{i}} X$. By Lemma 2.5 this means that, in the $x_{i}$-adapted orthogonal basis, $f-g$ is a linear combination of monomials whose gcds with $x_{i}$ have degrees at most $D-2$. Hence, by Lemma 2.4, $\left[f-g \mid x_{i}\right]_{\ell}=0$ for all $\ell=1, \ldots, p$. We conclude that, for each $\ell$,

$$
[f-g \mid g]_{\ell}=\sum_{i=1}^{k}\left[f-g \mid x_{i}\right]_{\ell}=0,
$$


and therefore

$$
[f \mid g]_{\ell}=[f-g \mid g]_{\ell}+[g \mid g]_{\ell}=0+0,
$$

where in the last step we used that [.|. $]_{\ell}$ is skew-symmetric. Hence, $g \in H_{f}$.

In the next section, we compute the dimension of the space spanned by the critical rank-one tensors for a general tensor and show that this space equals $H_{f}$.

\section{The scheme of critical rank-one tensors}

\subsection{Critical rank-one tensors as the zero locus of a vector bundle section}

Let $f \in T=\bigotimes_{\ell=1}^{p} S^{d_{\ell}} V_{\ell}$ be a tensor. We assume that $p \geq 2, d_{\ell} \geq 1$, and $\operatorname{dim} V_{\ell}=$ $n_{\ell}+1 \geq 1$ for all $\ell$. We adapt the notation of [15, Section 5.1] to our current setting.

Consider the Segre-Veronese variety $\mathbb{P} X=\mathbb{P} V_{1} \times \ldots \times \mathbb{P} V_{p}$ embedded with $\mathcal{O}\left(d_{1}, \ldots, d_{p}\right)$ in $\mathbb{P} T$; so $\mathbb{P} X$ is the projective variety associated with the affine cone $X \subseteq T$. Let $\pi_{\ell}: \mathbb{P} X \rightarrow \mathbb{P} V_{\ell}$ be the projection on the $\ell$ th factor and set $N:=\operatorname{dim} \mathbb{P} X=$ $n_{1}+\ldots+n_{p}$. For each $\ell \in[p]$ let $\mathcal{Q}_{\ell}$ be the quotient bundle on $\mathbb{P} V_{\ell}$, whose fiber over a point $\langle v\rangle$ is $V_{\ell} /\langle v\rangle$. From these quotient bundles, we construct the following vector bundles on $\mathbb{P} X$ :

$$
\mathcal{E}:=\bigoplus_{\ell=1}^{p} \mathcal{E}_{l} \quad \text { where } \quad \mathcal{E}_{l}:=\left(\pi_{\ell}^{*} \mathcal{Q}_{\ell}\right) \otimes \mathcal{O}\left(d_{1}, \ldots, d_{\ell-1}, d_{\ell}-1, d_{\ell+1}, \ldots, d_{p}\right)
$$

Note that $\mathcal{E}$ has $\operatorname{rank} N$. The fiber of $\mathcal{E}_{\ell}$ over a point $v:=\left(\left\langle v_{1}\right\rangle, \ldots,\left\langle v_{p}\right\rangle\right) \in \mathbb{P} X$ consists of polynomial maps $\prod_{i=1}^{p}\left\langle v_{i}\right\rangle \rightarrow V_{\ell} /\left\langle v_{\ell}\right\rangle$ that are multi-homogeneous of multi-degree $\left(d_{1}, \ldots, d_{\ell}-1, \ldots, d_{p}\right)$. The tensor $f$ yields a global section of $\mathcal{E}_{\ell}$ which over the point $v$ is the map sending $\left(c_{1} v_{1}, \ldots, c_{p} v_{p}\right)$ to the natural pairing of $f$ with $\left(c_{1} v_{1}\right)^{d_{1}} \cdots\left(c_{\ell} v_{\ell}\right)^{d_{\ell}-1} \cdots\left(c_{p} v_{p}\right)^{d_{p}}$-a vector in $V_{\ell}$-taken modulo $\left\langle v_{\ell}\right\rangle$. Combining these $p$ sections, $f$ yields a global section $s_{f}$ of $\mathcal{E}$. By Proposition 2.6, for $f$ sufficiently general, the tensor $x:=v_{1}^{d_{1}} \otimes \cdots \otimes v_{p}^{d_{p}}$ is a nonzero scalar multiple of a critical rank-one tensor for $f$ if and only if the point $\left(\left\langle v_{1}\right\rangle, \ldots,\left\langle v_{p}\right\rangle\right)$ is in the zero locus $Z_{f}$ of the section $s_{f}$. In [5], this is used to compute the cardinality of $Z_{f}$ for $f$ sufficiently general as the top Chern class of $\mathcal{E}$. Our current task is different: we want to show that, if we assume the triangle inequalities of Theorem 1.1 and that $f$ is sufficiently general, the linear span $\left\langle Z_{f}\right\rangle$ equals the projectivized critical space $\mathbb{P} H_{f}$; this is the second part of Theorem 1.1.

\subsection{Bott's formulas and a consequence}

Our central tool will be the following formulas for the cohomology of vector bundles over projective spaces [13]. Recall that $\Omega_{\mathbb{P}^{n}}^{r}(k)$ is the $\mathcal{O}(k)$-twisted sheaf of differential $r$-forms on $\mathbb{P}^{n}$.

Lemma 3.1 (Bott's formulas) For $q, n, r \in \mathbb{Z}_{\geq 0}$ and $k \in \mathbb{Z}$, we have

$$
h^{q}\left(\mathbb{P}^{n}, \Omega_{\mathbb{P}^{n}}^{r}(k)\right)= \begin{cases}\left(\begin{array}{c}
k+n-r \\
k
\end{array}\right)\left(\begin{array}{c}
k-1 \\
r
\end{array}\right) & \text { if } q=0 \leq r \leq n \text { and } k>r, \\
1 & \text { if } 0 \leq q=r \leq n \text { and } k=0, \\
\left(\begin{array}{c}
k+r \\
-k
\end{array}\right)\left(\begin{array}{c}
-k-1 \\
n-r
\end{array}\right) & \text { if } q=n \geq r \geq 0 \text { and } k<r-n, \text { and } \\
0 & \text { otherwise. }\end{cases}
$$

A consequence featuring the triangle inequalities of Theorem 1.1 is the following. 
Lemma 3.2 Suppose that $n_{\ell} \leq \sum_{i \neq \ell} n_{i}$ holds for all $\ell$ with $d_{\ell}=1$. Let $k \geq 2$ be an integer, $q_{1}, \ldots, q_{p}$ be nonnegative integers with $\sum_{\ell=1}^{p} q_{\ell}<k$ and $r_{1}, \ldots, r_{p}$ be nonnegative integers with $\sum_{\ell=1}^{p} r_{\ell}=k$. Then,

$$
\bigotimes_{\ell=1}^{p} H^{q_{\ell}}\left(\mathbb{P} V_{\ell}, \Omega_{\mathbb{P} V_{\ell}}^{r_{\ell}}\left(-d_{\ell}(k-1)+2 r_{\ell}\right)\right)=0 .
$$

Proof Assume that all factors in the tensor product are nonzero.

First, if all of the factors were nonzero by virtue of the second and third line in Bott's formulas, then we would have $q_{\ell} \geq r_{\ell}$ for all $\ell$, and hence $k>\sum_{\ell} q_{\ell} \geq \sum_{\ell} r_{\ell}=k$, a contradiction.

So some factor is nonzero by virtue of the first line in Bott's formulas; without loss of generality this is the first factor. Hence we have $q_{1}=0 \leq r_{1} \leq n_{1}$ and $-d_{1}(k-1)+2 r_{1}>r_{1}$. This last inequality reads $r_{1}>d_{1}(k-1)$. Combining this with $\sum_{\ell} r_{\ell}=k$ and the fact that $d_{1}$ is a positive integer, we find that $r_{1}=k, d_{1}=1$, and $r_{\ell}=0$ for $\ell>1$. In particular, there are no $\ell>1$ for which the first line in Bott's formulas applies.

For any $\ell>1$, if the second line applies, then $0=r_{\ell}=q_{\ell}=-d_{\ell}(k-1)+2 r_{\ell}$, which contradicts that both $d_{\ell}$ and $k-1$ are positive. Hence, the third line applies for all $\ell>1$, and in particular we have $q_{\ell}=n_{\ell}$. But then

$$
n_{1} \geq r_{1}=k>\sum_{l=1}^{p} q_{l}=\sum_{l=2}^{p} n_{l}
$$

which together with $d_{1}=1$ contradicts the triangle inequality in the lemma.

\subsection{Vanishing cohomology}

The vanishing result in this subsection uses Lemma 3.2 and the following version of Künneth's formula.

Lemma 3.3 (Künneth's formula) For vector bundles $\mathcal{G}_{\ell}$ on $\mathbb{P} V_{\ell}$ for $\ell=1, \ldots, p$ and a nonnegative integer $q$ we have

$$
H^{q}\left(\mathbb{P} X, \bigotimes_{\ell} \pi_{\ell}^{*} \mathcal{G}_{\ell}\right) \cong \bigoplus_{q_{1}+\ldots+q_{p}=q} \bigotimes_{\ell} H^{q_{\ell}}\left(\mathbb{P} V_{\ell}, \mathcal{G}_{\ell}\right),
$$

where the sum is over all p-tuples of nonnegative integers summing to $q$.

Lemma 3.4 Suppose that $n_{\ell} \leq \sum_{i \neq \ell} n_{i}$ holds for all $\ell$ such that $d_{\ell}=1$. Let $k \geq 2$ be an integer and $q \in\{0, \ldots, k-1\}$. Then, we have

$$
H^{q}\left(\mathbb{P} X,\left(\bigwedge^{k} \mathcal{E}^{*}\right) \otimes \mathcal{O}\left(d_{1}, \ldots, d_{p}\right)\right)=0 .
$$

Proof First,

$$
\mathcal{E}^{*}=\bigoplus_{\ell=1}^{p}\left(\pi_{\ell}^{*} \mathcal{Q}_{\ell}{ }^{*}\right) \otimes \mathcal{O}\left(-d_{1}, \ldots,-d_{\ell-1},-\left(d_{\ell}-1\right),-d_{\ell+1}, \ldots,-d_{p}\right) .
$$

A well-known formula for $k$ th wedge power of a direct sum yields

$$
\bigwedge^{k} \mathcal{E}^{*}=\bigoplus_{r_{1}+\ldots+r_{p}=k} \bigotimes_{\ell} \bigwedge^{r_{\ell}}\left(\pi_{\ell}^{*} \mathcal{Q}_{\ell}{ }^{*} \otimes \mathcal{O}\left(-d_{1}, \ldots,-\left(d_{\ell}-1\right), \ldots,-d_{p}\right)\right) .
$$


Using $\bigwedge^{r}(\mathcal{F} \otimes \mathcal{O}(\omega))=\left(\bigwedge^{r} \mathcal{F}\right)(r \omega), \mathcal{Q}^{*}=\Omega^{1}(1)$, and $\bigwedge^{r}\left(\Omega^{1}(1)\right)=\Omega^{r}(r)$, we obtain

$$
\bigwedge^{k} \mathcal{E}^{*}=\bigoplus_{r_{1}+\ldots+r_{p}=k} \bigotimes\left(\pi_{\ell}^{*} \Omega_{\mathbb{P} V_{\ell}}^{r_{\ell}}\left(r_{\ell}\right) \otimes \mathcal{O}\left(-r_{\ell} d_{1}, \ldots,-r_{\ell}\left(d_{\ell}-1\right), \ldots,-r_{\ell} d_{p}\right) .\right.
$$

Twisting by $\mathcal{O}\left(d_{1}, \ldots, d_{p}\right)$, regrouping in each projection, and using $\sum_{\ell} r_{\ell}=k$ we find:

$$
\left(\bigwedge^{k} \mathcal{E}^{*}\right) \otimes \mathcal{O}\left(d_{1}, \ldots, d_{p}\right)=\bigoplus_{r_{1}+\ldots+r_{p}=k} \bigotimes_{\ell} \pi_{\ell}^{*} \Omega_{\mathbb{P} V_{\ell}}^{r_{\ell}}\left(-d_{\ell}(k-1)+2 r_{\ell}\right) .
$$

To compute $H^{q}$ of each summand we apply Künneth's formula, and obtain subsummands which are exactly of the form in Lemma 3.2, hence zero.

\subsection{Comparing $\mathbb{P} H_{f}$ and $\left\langle Z_{f}\right\rangle$}

Assume that $f$ is sufficiently general in $T$. By the first subsection of this section and by Proposition 2.6, $Z_{f}$ is contained in the projectivized critical space $\mathbb{P} H_{f}$, hence so is $\left\langle Z_{f}\right\rangle$. Our goal now is to show that $\left\langle Z_{f}\right\rangle$ is equal to $\mathbb{P} H_{f}$ and to compute its dimension. Both of these goals are achieved through the following lemma. The section $s_{f}$ of $\mathcal{E}$ yields a homomorphism $\mathcal{E}^{*} \rightarrow \mathcal{O}$ of sheaves whose image is contained in the ideal sheaf $\mathcal{I}_{Z_{f}}$ of the zero locus of $s_{f}$.

Lemma 3.5 Assume that for each $\ell \in[p]$ we have $n_{\ell} \leq \sum_{i \neq \ell} n_{i}$ and that $f$ is sufficiently general. Then, the induced homomorphism $\mathcal{E}^{*} \otimes \mathcal{O}\left(d_{1}, \ldots, d_{p}\right) \rightarrow \mathcal{I}_{Z_{f}} \otimes \mathcal{O}\left(d_{1}, \ldots, d_{p}\right)$ induces an isomorphism at the level of global sections.

The following proof can be shortened considerably using spectral sequences, but we found it more informative in its current form. To make the formulas more transparent, we write $H^{q}($.$) instead of H^{q}(\mathbb{P} X,$.$) everywhere.$

Proof To establish the desired isomorphism

$$
H^{0}\left(\mathcal{E}^{*} \otimes \mathcal{O}\left(d_{1}, \ldots, d_{p}\right)\right) \cong H^{0}\left(\mathcal{I}_{Z_{f}} \otimes \mathcal{O}\left(d_{1}, \ldots, d_{p}\right)\right)
$$

we use the following Koszul complex (see, e.g., [8, Chapter III,Proposition 7.10A]):

$$
0=\bigwedge^{N+1} \mathcal{E}^{*} \rightarrow \bigwedge^{N} \mathcal{E}^{*} \rightarrow \cdots \rightarrow \bigwedge^{2} \mathcal{E}^{*} \rightarrow \mathcal{E}^{*} \rightarrow \mathcal{I}_{Z} \rightarrow 0
$$

Letting $\mathcal{F}_{k}$ be the quotient of $\bigwedge^{k} \mathcal{E}^{*}$ by the image of $\bigwedge^{k+1} \mathcal{E}^{*}$, this yields the short exact sequence

$$
0 \rightarrow \mathcal{F}_{2} \rightarrow \mathcal{E}^{*} \rightarrow \mathcal{I}_{Z} \rightarrow 0 .
$$

Tensoring with $\mathcal{O}\left(d_{1}, \ldots, d_{p}\right)$ yields the short exact sequence

$$
0 \rightarrow \mathcal{F}_{2} \otimes \mathcal{O}\left(d_{1}, \ldots, d_{p}\right) \rightarrow \mathcal{E}^{*} \otimes \mathcal{O}\left(d_{1}, \ldots, d_{p}\right) \rightarrow \mathcal{I}_{Z} \otimes \mathcal{O}\left(d_{1}, \ldots, d_{p}\right) \rightarrow 0,
$$

and this gives a long exact sequence in cohomology beginning with

$$
\begin{aligned}
0 & \rightarrow H^{0}\left(\mathcal{F}_{2} \otimes \mathcal{O}\left(d_{1}, \ldots, d_{p}\right)\right) \rightarrow H^{0}\left(\mathcal{E}^{*} \otimes \mathcal{O}\left(d_{1}, \ldots, d_{p}\right)\right) \rightarrow H^{0}\left(\mathcal{I}_{Z} \otimes \mathcal{O}\left(d_{1}, \ldots, d_{p}\right)\right) \\
& \rightarrow H^{1}\left(\mathcal{F}_{2} \otimes \mathcal{O}\left(d_{1}, \ldots, d_{p}\right)\right) \rightarrow
\end{aligned}
$$

So to obtain the desired isomorphism we want that

$$
H^{q}\left(\mathcal{F}_{2} \otimes \mathcal{O}\left(d_{1}, \ldots, d_{p}\right)\right)=0 \quad \text { for } q=0,1 .
$$


For each $k=2, \ldots, N$, we have the short exact sequence

$$
0 \rightarrow \mathcal{F}_{k+1} \rightarrow \bigwedge^{k} \mathcal{E}^{*} \rightarrow \mathcal{F}_{k} \rightarrow 0
$$

which yields the long exact sequence

$$
\begin{aligned}
& \rightarrow H^{k-2}\left(\bigwedge^{k} \mathcal{E}^{*} \otimes \mathcal{O}\left(d_{1}, \ldots, d_{p}\right)\right) \rightarrow H^{k-2}\left(\mathcal{F}_{k} \otimes \mathcal{O}\left(d_{1}, \ldots, d_{p}\right)\right) \\
& \rightarrow H^{k-1}\left(\mathcal{F}_{k+1} \otimes \mathcal{O}\left(d_{1}, \ldots, d_{p}\right)\right) \rightarrow H^{k-1}\left(\bigwedge^{k} \mathcal{E}^{*} \otimes \mathcal{O}\left(d_{1}, \ldots, d_{p}\right)\right) \\
& \rightarrow H^{k-1}\left(\mathcal{F}_{k} \otimes \mathcal{O}\left(d_{1}, \ldots, d_{p}\right)\right) \rightarrow H^{k}\left(\mathcal{F}_{k+1} \otimes \mathcal{O}\left(d_{1}, \ldots, d_{p}\right)\right) \rightarrow
\end{aligned}
$$

Using Lemma 3.4, the two leftmost spaces are zero, so that

$$
\begin{aligned}
& H^{k-2}\left(\mathcal{F}_{k} \otimes \mathcal{O}\left(d_{1}, \ldots, d_{p}\right)\right) \cong H^{k-1}\left(\mathcal{F}_{k+1} \otimes \mathcal{O}\left(d_{1}, \ldots, d_{p}\right)\right) \text { and } \\
& H^{k-1}\left(\mathcal{F}_{k} \otimes \mathcal{O}\left(d_{1}, \ldots, d_{p}\right)\right) \subseteq H^{k}\left(\mathcal{F}_{k+1} \otimes \mathcal{O}\left(d_{1}, \ldots, d_{p}\right)\right)
\end{aligned}
$$

Hence, using that $\mathcal{F}_{N+1}=0$, we find that

$$
\begin{aligned}
& H^{0}\left(\mathcal{F}_{2} \otimes \mathcal{O}\left(d_{1}, \ldots, d_{p}\right)\right) \cong \cdots \cong H^{N-1}\left(\mathcal{F}_{N+1} \otimes \mathcal{O}\left(d_{1}, \ldots, d_{p}\right)\right)=0 \text { and } \\
& H^{1}\left(\mathcal{F}_{2} \otimes \mathcal{O}\left(d_{1}, \ldots, d_{p}\right)\right) \subseteq \cdots \subseteq H^{N}\left(\mathcal{F}_{N+1} \otimes \mathcal{O}\left(d_{1}, \ldots, d_{p}\right)\right)=0
\end{aligned}
$$

as desired.

Proposition 3.6 Suppose that for each $\ell \in[p]$ we have $n_{\ell} \leq \sum_{i \neq \ell} n_{i}$ and that $f$ is sufficiently general. Then, $\left\langle Z_{f}\right\rangle=\mathbb{P} H_{f}$ and $\operatorname{codim}_{T} H_{f}=\sum_{\ell}\left(\begin{array}{c}n_{\ell}+1 \\ 2\end{array}\right)$.

Proof Since $\mathbb{P} X$ is embedded by $\mathcal{O}\left(d_{1}, \ldots, d_{p}\right)$, the space of linear forms on $T$ vanishing on $Z_{f}$ is $H^{0}\left(\mathcal{I}_{Z_{f}} \otimes \mathcal{O}\left(d_{1}, \ldots, d_{p}\right)\right)$. By Lemma 3.5 , this space is isomorphic to

$$
\begin{aligned}
& H^{0}\left(\mathcal{E}^{*} \otimes \mathcal{O}\left(d_{1}, \ldots, d_{p}\right)\right)=\bigoplus_{\ell} H^{0}\left(\pi_{\ell}^{*} \mathcal{Q}_{l}^{*} \otimes \mathcal{O}(0, \ldots, 1, \ldots, 0)\right) \\
& =\bigoplus_{\ell} H^{0}\left(\pi_{\ell^{*}}\left(\Omega_{\mathbb{P} V_{\ell}}^{1}(2)\right)\right)=\bigoplus_{\ell} H^{0}\left(\mathbb{P} V_{\ell}, \Omega_{\mathbb{P} V_{\ell}}^{1}(2)\right)
\end{aligned}
$$

which by the first line in Bott's formulas has dimension $\sum_{\ell}\left(\begin{array}{c}n_{\ell}+1 \\ 2\end{array}\right)$. This means that $\operatorname{codim}_{\mathbb{P} T}\left\langle Z_{f}\right\rangle=\sum_{\ell}\left(\begin{array}{c}n_{\ell}+1 \\ 2\end{array}\right)$, so the second statement in the proposition follows from the first statement.

To establish the first statement, we spell out the map

$$
H^{0}\left(\mathbb{P} V_{\ell}, \mathcal{Q}_{\ell}^{*} \otimes \mathcal{O}(1)\right)=H^{0}\left(\mathbb{P} V_{\ell}, \Omega_{\mathbb{P} V_{\ell}}^{1}(2)\right) \rightarrow H^{0}\left(\mathcal{I}_{Z_{f}} \otimes \mathcal{O}\left(d_{1}, \ldots, d_{p}\right)\right)
$$

in greater detail. The space on the left is canonically $\left(\bigwedge^{2} V_{\ell}\right)^{*}$, and an element $\xi$ in this space is mapped to the linear form $T \rightarrow \mathbb{C}, g \mapsto \xi\left([f \mid g]_{\ell}\right)$. As $\ell$ varies, these are precisely the linear forms that cut out $H_{f}$. This proves that $\mathbb{P} H_{f}=\left\langle Z_{f}\right\rangle$. 
Remark 3.7 In general, for the equality $\left\langle Z_{f}\right\rangle=\mathbb{P} H_{f}$ we only need that the linear equations cutting out $\mathbb{P} H_{f}$ also cut out $Z_{f}$, i.e., we only need that the linear map in Lemma 3.5 is surjective. One might wonder whether this surjectivity remains true when the triangle inequalities fail. In the case of $\left(n_{1}+1\right) \times\left(n_{2}+1\right)$-matrices, it does indeed-there we already knew the critical rank-one approximations span the critical space-but for $p=3$ and $2 \times 2 \times 4$-tensors (so that $n_{3}=3>1+1=n_{1}+n_{2}$ ) the space $\left\langle Z_{f}\right\rangle$ has dimension 6 while computer experiments suggest that the space $\mathbb{P} H_{f}$ has dimension 7 , hence the surjectivity fails. Still, in these experiments, $f$ itself seems to lie in the span of $Z_{f}$. This leads to the open problem whether our analogue of the Spectral Theorem and the Eckart-Young Theorem persists when the triangle inequalities fail.

\section{Proofs of the main results}

Proof of Theorem 1.1 The first statement is Proposition 2.12; the second and third statement are Proposition 3.6. The last statement follows from Remark 2.9.

Proof of Corollaries 1.2 and 1.3. If $g$ is a real tensor of real rank at most $k$ closest to $f$, then one can write it as $x_{1}+\cdots+x_{k}$ with $x_{1}, \ldots, x_{k}$ real points of $X$. In particular, all of these points are non-isotropic, and the argument of Proposition 2.12 applies. Hence, $g$ lies in $H_{f}$. Now the result follows from Proposition 3.6. The argument applies, in particular, to $k$ equal to the rank of $f$, which gives the last statement of the corollaries.

Note that, if $f$ is any real tensor, then any real tensor of real rank at most $k$ closest to $f$ lies in $H_{f}$ by the argument above. Only for the conclusion that it lies in the span of the complex critical rank-one tensors of $f$ do we use that $f$ is sufficiently general. We do not know whether this generality is really needed.

Also note that we do not shed new light on the question of when for sufficiently general $f$ there exists a closest real tensor of rank at most $k$. For an update on the complex case, see [17].

\footnotetext{
Author details

${ }^{1}$ Mathematisches Institut, Universität Bern, Sidlerstrasse 5, 3012 Bern, Switzerland, ${ }^{2}$ Department of Mathematics and Computer Science, Eindhoven University of Technology, Eindhoven, The Netherlands, ${ }^{3}$ Dipartimento di Matematica e Informatica U. Dini, Università di Firenze, viale Morgagni 67/A, 50134 Florence, Italy, ${ }^{4}$ Departamento de Álgebra, Geometría y Topología, Universidad de Málaga, Bulevar Louis Pasteur, 31, 29010 Málaga, Spain.
}

Received: 30 November 2017 Accepted: 9 May 2018 Published online: 23 May 2018

\footnotetext{
References

1. Boralevi, A., Draisma, J., Horobeţ, E., Robeva, E.: Orthogonal and unitary tensor decomposition from an algebraic perspective. Isr. J. Math. 222, 223-260 (2017)

2. de Silva, V., Lim, L.-H.: Tensor rank and the ill-posedness of the best low-rank approximation problem. SIAM J. Matrix Anal. Appl. 30, 1084-1127 (2008)

3. Draisma, J., Horobeţ, E., Ottaviani, G., Sturmfels, B., Thomas, R.: The Euclidean distance degree of an algebraic variety. Found. Comput. Math. 16(1), 99-149 (2016)

4. Drusvyatskiy, D., Lee, H.-L., Ottaviani, G., Thomas, R.: The Euclidean distance degree of orthogonally invariant matrix varieties. Isr. J. Math 221, 291-316 (2017)

5. Friedland, S., Ottaviani, G.: The number of singular vector tuples and uniqueness of best rank one approximation of tensors. Found. Comput. Math. 14(6), 1209-1242 (2014)

6. Friedland, S., Tammali, V.: Low-rank approximation of tensors. In: Benner, P., Bollhöfer, M., Kressner, D., Mehl, C., Stykel, T. (eds.) Numerical Algebra, Matrix Theory, Differential-Algebraic Equations and Control Theory. Springer, Cham (2015)

7. Gelfand, I., Kapranov, M., Zelevinsky, A.: Discriminants, Resultants and Multidimensional Determinants. Birkhäuser, Boston (1994)

8. Hartshorne, R.: Algebraic Geometry, Graduate Texts in Mathematics 52. Springer, New York (1977)

9. Landsberg, J.M.: Tensors: Geometry and Applications, Volume 128 of Graduate Studies in Mathematics. American Mathematical Society (AMS), Providence (2012)
} 
10. Lim, L.H.: Singular values and eigenvalues of tensors: a variational approach. In: Proceedings of IEEE International Workshop on Computing Advances in Multi-sensor Adaptive Processing (CAMSAP 2005), pp. 129-132

11. Maccioni, M.: The number of real eigenvectors of a real polynomial, to appear in Bollettino dell'Unione Matematica Italiana. arXiv:1606.04737

12. Mirsky, L.: Symmetric gauge functions and unitarily invariant norms. Q. J. Math. Oxf. II Ser. 11, 50-59 (1960)

13. Okonek, C., Schneider, M., Spindler, H.: Vector Bundles on Complex Projective Spaces, Progress in Mathematics, vol. 3. Birkhäuser, Boston (1980)

14. Oeding, L., Ottaviani, G.: Eigenvectors of tensors and algorithms for waring decomposition. J. Symb. Comput. 54, 9-35 (2013)

15. Ottaviani, G., Paoletti, R.: A geometric perspective on the singular value decomposition. Rend. Istit. Mat. Univ. Trieste 47, 107-125 (2015)

16. Ottaviani, G., Tocino, A.: Best rank k approximation for binary forms. Collect. Math. 69, 163-171 (2018)

17. Qi, Y., Michałek, M., Lim, L.H.: Complex tensors almost always have best low-rank approximations. preprint. arXiv:1711.11269

18. Vannieuwenhoven, N., Nicaise, J., Vandebril, R., Meerbergen, K.: On generic nonexistence of the Schmidt-EckartYoung decomposition for complex tensors. SIAM J. Matrix Anal. Appl. 35(3), 886-903 (2014) 